\title{
Preprocessing Medical Images for Classification using Deep Learning Techniques
}

\author{
A.Rama, A.Kumaravel, C. Nalini
}

\begin{abstract}
Recently, the demand for computer vision techniques is continuously rising because of the development of techniques in decision making pertaining to health sector. Image processing is a subset of computer vision which makes use of algorithms to perform vision emulation to recognize objects. In this study a novel convolutional neural network is configured based on deep learning to classifying Chest $x$-ray images into five major classes. It addresses an issue of insufficiency in medical images for employing deep learning for image classification. A new augmentation technique superimposing of images helps to generate more new samples from the available images using label-preserving transformations. Data augmentation technique can generate new sample data from the original data using various transforming strategies. Therefore the data augmentation technique helps in accumulating enough data for processing to obtain better performance. The main objective of superimposing of two images is to minimize redundancy and uncertainty in the output image. Therefore the superimposing carried out with original image and a set of various augmented image to obtain better accuracy. Later results of various superimposing techniques are compared and evaluated to demonstrate the better techniques. It is concluded that the proposed techniques can obtain better performance in medical image classification problem.
\end{abstract}

Keywords: Classification, flip, transform, augmentation, superimposing.

\section{INTRODUCTION}

Deep learning methods got greatly progressed in medical image diagnosing, currently, most of the deep learning model got greatly progressed in medical image classification. As deep learning model are in need of large data set for training , the main problem occurs are availability of enough data for training. To increase the image availability an effective preprocessing technique called data augmentation technique has been proposed. Data augmentation technique can generate new sample data from the original data using various transforming strategies. Therefore the data augmentation technique helps in accumulating enough data for processing to obtain better performance. In this work various data augmentation techniques have been proposed to increase performance in image classification. There are various image augmentation strategies that include

Revised Manuscript Received on July 22, 2019.

A.Rama Assistant Professor, Department of Information Technology, Bharath Institute of Higher Education and Research, Chennai, India. Email-rama_j1@gmail.com

Dr.A.Kumaravel, Professor, Department of Information Technology, Bharath Institute of Higher Education and Research, Chennai, India Email - drkumaravel@gmail.com

Dr. C. Nalini, Assistant Professor, Department of Information Technology, Bharath Institute of Higher Education and Research, Chennai, India. Email nalinicha2002@gmail.com horizontal and vertical flipping, cropping, jitter, scaling, rotate, transform etc... In this work a new data augmentation strategies have been proposed where a new sample of data can be obtained from overlaying two images called superimposing of images [11]. For superimposing, the images are selected randomly from the data set and it will be the original image, the selected images are augmented by applying any one of the augmentation techniques. The augmented image is overlaid on its respective original image, therefore to obtain the superimposed images. Superimposing image is a method of merging the significant information from a two images into a single image, thus the resulting superimposed image will consist of more absolute and informative of than the original input image. There are few significant requirements while superimposing two images. The superimposed images should retain all of its relevant feature information from the input images. The superimposing technique should give more accurate and appropriate diagnosis results. The main objective of superimposing of two images is to minimize redundancy and uncertainty in the output image. Therefore the superimposing carried out with original image and a set of various augmented image to obtain better accuracy. The main advantage data augmentation technique is to generate more amounts of synthetic data from real data, therefore to avoid under fitting while training the Classifier.

\section{LITERATURE RVIEW}

Mayan Frid-Adar et al), [10] recommended another strategy for information enlargement, which can make new pictures with the assistance of Generative Adversarial Networks (GANs). The quantity of preparing pictures got expanded by actualizing run of the mill information growth strategies. It amplifies the size of information with decent variety by executing GAN strategies, accordingly to prepare the classifier. The proposed information increase systems get fundamentally improved affectability to $85.7 \%$ and $92.4 \%$ of explicitness. (Sebastian C. Wong, et al), [12] suggested the reason and points of interest of expansions. AI classifier are utilized for preparing the enlarging the information. Two methodologies are actualized for expanding the quantity of preparing tests, first methodology is information are distorted and it can produces all the more preparing tests as the information space got changed. Later it is assessed tentatively to consider the advantages of the proposed information expansion methods dependent on transcribed digit MNIST dataset. Three sorts of designs are utilized for assessing the enlargement procedure. The primary engineering is convolutional back 
engendering prepared neural system; the second is convolutional SVM and third is convolutional AI classifier. The outcome demonstrates that increase in information space offers a superior bit of leeway for upgrading the exhibition and limiting the overfitting.

(Zeshan Hussain, Francisco Gimenez et al) proposes a different growth procedure to discover the effective information expansion to catch the insights of therapeutic pictures, which can prompt new discriminative models. The creator executed different enlargement procedures and showed that to the degree preparing set which expanded can perform better. All the expansion procedures are executing by holding the first picture properties, later the prepared and assessed yields are contrasted with decide the better growth methodologies. In general, the enlargement strategies that incredibly affect segregate execution and furthermore seriously affect generative execution.

(Avinash Kori et al), [7] prescribed novel information enlargement strategies dependent on Position Coordinates to advance execution in picture order utilizing different imaging applications. It utilizes the pixel directions of a picture for increase thusly to accomplish better execution by goals invariant. The creator demonstrated that the system expressed had advanced in characterization precision when contrasted with different Neural Network classifier models that are prepared without nearby pixel arranges. The usage results demonstrate that the position directions of an assistant info acquire better precision results just as improved mutliresolution execution. Alhussein Fawzi proposed another calculation that can change preparing tests consequently to new enlarged information [7]. The significant reason for existing is to look for moment change to yield least characterization blunder. Later the incorporated increase strategies alongside SGD ('Stochastic Gradient Descent') calculation for playing out the DNN. The analyses are exhibited utilizing two datasets and demonstrated that the strategy proposed is a straightforward plan that can acquire more prominent outcomes to propel strategies. Ling Ma Xiabi Liuet al proposes CBMIR (Content Based Medical Image Retrieval), [8] by intertwining challenge touchy similitude. Pictures in the predetermined dataset are intertwined with inquiry picture thinking about the closeness and semantic. In this manner, weighted diagrams are work with the pictures that are spoken to as hubs while the likenesses as edges. Most limited way calculation is utilized to register the weighted diagram along these lines to get similitude measure. The recovery framework achieved by comparability measures between the inquiry picture and the predefined database picture are figured. The semantic hole got diminished by the comparability measures to achieve the similitude of the setting delicate for a superior recovery execution. The new method is assessed with other recovery framework utilizing the Common Signs of Lung Diseases Imaging (CISLs) and demonstrated by accomplishing improved outcomes in recovery and calculation execution.

A proficient framework dependent on CBMIR is proposed in particular FCSS recovers Common Signs of Lung Diseases Imaging (CISLs) is to intertwine the similitude and measure it precisely dependent on closeness of setting touchy which aides in advancing the exhibition of the recovery framework [2]. The strategy for melding semantics and visual comparability simultaneously by the pair insightful likeness help to lessen the semantic hole and to persevere through wrong order. In assessment with other recovery framework the strategy got from the blend of both visual comparability and various leveled perform better. The calculation to be specific most limited way helps for restrict different picture information over the diagram. To finish the whole recovery strategy, the lengths of briefest ways are utilized for figuring likenesses of setting touchy of the inquiry pictures [2]. The aftereffects of the investigation showed that the new procedure performs superior to anything other recovery strategy by overlooking the structure of the information complex. The FCSS is executing to recover CISLs. As CISLs are identified with lung maladies, it helps in determination different lung sicknesses. It helps for recovering comparative CISLs from authentic CT sweeps and returns the related data for $\mathrm{CAD}$. The pictures in the database are gathered to shape bunch in this way to create a diagram. The running time for the most brief way calculation is decreased by actualizing it on a huge database. Also, the single marked information that holds injury may display in a few CISLs is called multilabel information. A change handling to change the multi-named learning technique to single-named learning strategy is performed over and over to recover and combine the pictures with the last outcomes. Saman Sarraf [5] exhibited two powerful pipelines utilizing broad pre-handling modules and DL based classifiers of MRI and fMRI information for separating mind pictures that are influenced by Alzheimer's disorder from typical sound cerebrums in more established grown-ups. Essential invariant highlights were removed from an enormous picture dataset utilizing CNN design which results in a profoundly precise and reproducible prescient model [4]. The accomplished exactness rates utilizing MRI and fMRI modalities dependent on different designs like LeNet, GoogLeNet demonstrates to be better than every single past technique utilized. Besides, at first fMRI information are utilized to prepare a DL based pipelines. This effective and dynamic DL based structures can be ensnared for various applications by arranging cerebrum issue in both clinical preliminaries. It can likewise demonstrate that the made pipelines give a fruitful calculation in separating multimodal MRI. At last, the characterization is intended for restorative purposes that permit figuring in strength of the fMRI and MRI pipelines. Arrangement of typical mind picture and Alzheimer's pictures required couple of ventures from pre-preparing for acknowledgment, which are brought about the advancement to a start to finish pipeline. The acknowledgment pipeline happens in three stages. Initial step is to pre-preparing, where the subsequent advance is to change over information and the last advance is the order. Two different methodologies are utilized in the pre-handling steps. Later the pre-prepared information are changed over from picture to a lossless Portable Network Graphics (PNG) which is encouraged as contribution for the DL based 
classifier. At last, the $\mathrm{CNN}$ based design is prepared and tried utilizing $75 \%$ and $25 \%$ of the dataset separately. Two unique pipelines are made which was executed in pre-preparing steps however utilizes comparable information change and grouping steps. The proposed technique shows better execution.

Chuanhai Zhang, [8] prescribed an order procedure to determine the unbalancing issue in restorative imaging. It is incorporated to the essential named dataset by radiologist for further verification[5]. The examination demonstrates that the proposed method named Unified LF\&SM accomplish the most superb outcomes, by picking high level of prescribed applicable pictures to accomplish better execution in grouping. It can likewise be used for some other issues identified with medicinal picture arrangement. The proposed increase strategy proposed was significantly diminishing manual marking cost. This technique can widely diminish manual marking time. The creator showed the investigations utilizing Unified LF\&SM gets the best outcome. Choosing significant pictures with high rates are suggestion for accomplishing the best exactness in grouping task. It is extended to other therapeutic picture characterization issues.

\section{MATERIALS AND METHODS}

We have considered 2000 chest X-ray images, Initially 360 normal images and 700 images consisting of various Pathology images are saved in two folders that are labeled with their specific Pathology name [1]. The original images are chosen randomly and various augmentations are applied to generate multiple images and saved in different folders with name of the equivalent augmentation method. The total images used for the experiment are increased to 2000 after augmentation in which 1700 images are abnormal images having various pathology labeled with specific Pathology name and 300 images are healthy images. Finally, the input dataset is divided as training set and validation set, where $80 \%$ of the images are considered for the training set and the remaining as a validation set. The original input images are collected from the three open source datasets Chest X-ray8, JSRT and Shenzhen [8].

\section{EXPERIMENTAL PROCEDURES}

Experiments are performed over nine different augmented image set; the various augmented images are stored in different folders which are labeled by their corresponding augment name. Every augmented dataset consist of augmented images of various pathology and the normal images. The augmented images are again preprocessed for resizing it to $200 \mathrm{X} 200$ pixels. The experiments are executed repeatedly with various augmented image dataset for obtain any of the five classes

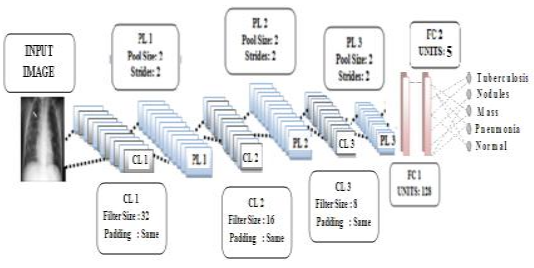

Figure 1: Novel configured CNN architecture

[1] A Novel construction of CNN consist of three convolutional layers with the parameters such as filer size as 32,16,8 for subsequent layers, activation function CReLU and padding type as same. It also have three pooling layers, where every convolutional layer are followed by a pooling layer with the parameter such as stride size as 2 , pooling type as max pooling and polling size 2 . These layers are further followed by two fully connected layers with the parameter such as optimization technique Adam, which performs gradient-based optimization and final Layer consisting of five nodes equal to the number of classes. The various augmentation techniques applied are described below

\section{A. Augmentation Method (Superimposing Of Images)}

This section illustrates the superimposing technique in data augmentation. In this method a new synthetic image is created by overlaying two images from the image data set. An approach of considering the average pixel intensity of two overlaid images is Synthesizing of images. For this approach a randomly selected input image as ImageX and the augmented image of ImageX as Image A. ImageX and Image A are superimposed to obtain final synthetic image ImageS. Later all the superimposed images along with the original input images are fed into the $\mathrm{CNN}$ for training.

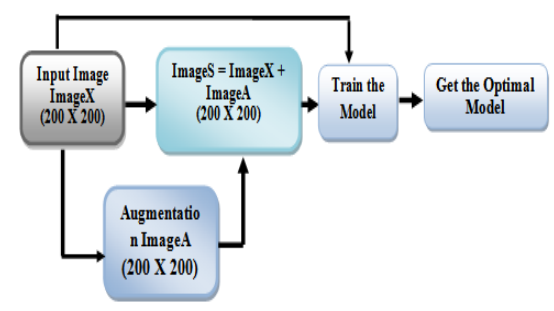

Figure 2: Overview of the augmentation techniques

While superimposing of images is used, both training set accuracy and validation set accuracy got enhanced. As various types of data augmentation are implemented the superimposing of images along with augmented image obtains better result. Image augmentation technique is proposed for generating more training data, therefore the original image and the augmented image both combined to increases the number of image used as the training set, it also helps in exploring the classifier to make it more robust. Different augmentations techniques are implemented using imgaug library

\section{B. Steps Involved In Superimposing Images}

Step 1: Load Input data

Step 2: Generate new samples data.

Step 3: Vary the parameters.

Step 4: Export the generated augmented images to their corresponding labeled folders.

Step 5: Repeat step3 and 4 for different augmentation type.

Step 6: superimpose augmented image using 
overlay function with their corresponding original image.

Step 7: Repeat step 4 for storing the superimposed images to their consequent labeled folders

Step 8: Train and validate the proposed CNN model

\section{Various Superimposing Techniques}

This section discusses about six different superimposing techniques used for the experiments. The original images are applied with five basic augmentations namely flip vertical, flip horizontal, zoom, rotate left and rotate right[6]. These various augmented images are overlaid on the original image to obtain five various superimposed images. Later these images are stored in different folders having the specific augmentation name. The augmented images along with the original images are trained and evaluated to get better accuracy. The augmentation techniques which obtain better accuracy results are identified.
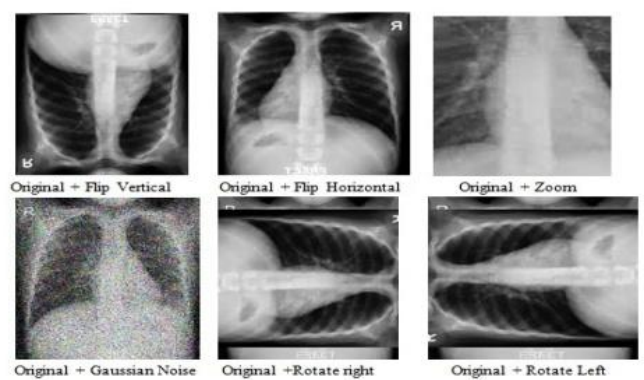

Figure 3: Sample Superimposed Images

We employ six various augmentation approaches to create six new training sets. Each newly generated training set consist of the original images and the augmented [1]. The augmentation techniques used for the experiments are as follows:

Superimpose original + Flip Horizontal: The original images are randomly chosen and they are applied with augmentation method Horizontal_flips by setting the parameter as true with various degree to transform the chosen images [1]. Those flipped images are overlaid on original image and saved in folder named horizontal flip.

Superimpose original + Flip Vertical: The original images are randomly chosen and they are applied with augmentation method Vertical_parameter is set as true with various degree to transform randomly chosen images from the original training set [1]. By setting the parameter as true with various degree to transform the chosen images [1]. Those flipped images are overlaid on original image and saved in folder named vertical flip.

Superimpose original + Rotation left: The original images are randomly chosen and they are applied with augmentation method Rotations. Rotations are applied with fewer angles; rotation_ranges parameter is set with value range between 160 degree and 90 degree. Those rotated images are overlaid on original image and saved in folders named rotation left.

Superimpose original + Rotation right: The original images are randomly chosen and they are applied with augmentation method Rotations. Rotations are applied with fewer angles; rotation_ranges parameter is set with value range between 0 degree and 90 degree [1]. . Those rotated images are overlaid on original image and saved separately in folders namely rotation left.

Superimpose original + Gaussian Noise: Images are selected randomly Gaussian Noise filter is applied and they are saved in folder named Gaussian.

Superimpose original + Shift (Height and Width): Images are randomly selected from the original dataset, then, for each chosen images are shifted with the value ranges between 0.3 to 0.5 respectively and they are overlaid on the original image and saved in separate folder named Shift.

Superimpose original + Shears: Vertical and horizontal shear are applied to the randomly selected images where the value of shear ranges between 0.2 to 0.5 respectively and they are saved in separate folder named Shear.

\section{TRAINING RESULTS AND DISCUSSIONS}

The experiments are executed using proposed CNN Architecture as demonstrated in fig1 and the setup shows better result

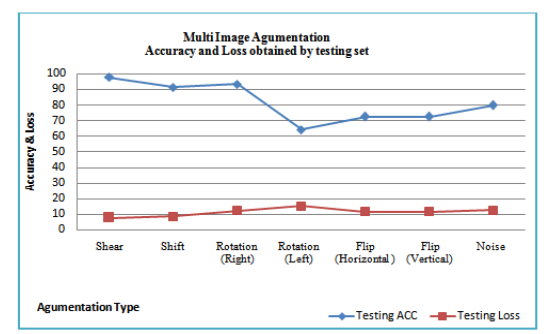

Figure 4- Accuracy and loss value for various augmented images

All the methods are implemented using superimposing of images with various augmentations. The accuracy rate and loss rate for various super imposing techniques are demonstrated as graphs in figure 4 the accuracies for the shear obtains the highest accuracy value 97.5.

\section{SUMMARY}

In this paper the deficiency of medical image classification using deep learning is addressed. Data augmentation technique using superimposing of original image with the corresponding augmented images helps in generating more new samples using label preserved transformations. In this work superimpose of augmented images over the corresponding original images are used for training therefore to capture the most important information, and they are applied I to the constructed classification model. . Later superimposing strategies which is best suitable for medical image classifications are identified. Here range of superimposing technique results are compared and evaluated to identify the better techniques. It is concluded that shear lead to validation accuracies of $97.7 \%$ and with loss value $0.9 \%$ is obtained where rotation gives the least accuracy of $63.7 \%$ of accuracy. 


\section{REFERENCES}

[1] Kumaravel A., Rangarajan K.,Algorithm for automaton specification for exploring dynamic labyrinths,Indian Journal of Science and Technology,V-6,I-SUPPL5,PP-4554-4559,Y-2013

[2]P. Kavitha, S. Prabakaran "A Novel Hybrid Segmentation Method with Particle Swarm Optimization and Fuzzy C-Mean Based On Partitioning the Image for Detecting Lung Cancer" International Journal of Engineering and Advanced Technology (IJEAT) ISSN: 2249-8958, Volume-8 Issue-5, June 2019

[3] Kumaravel A., Meetei O.N.,An application of non-uniform cellular automata for efficient cryptography,2013 IEEE Conference on Information and Communication Technologies, ICT 2013,V-,I-,PP-1200-1205,Y-2013

[4] Kumarave A., Rangarajan K.,Routing alogrithm over semi-regular tessellations,2013 IEEE Conference on Information and Communication Technologies, ICT 2013,V-,I-,PP-1180-1184,Y-2013

[5]P. Kavitha, S. Prabakaran "Designing a Feature Vector for Statistical Texture Analysis of Brain Tumor" International Journal of Engineering and Advanced Technology (IJEAT) ISSN: 2249-8958, Volume-8 Issue-5, June 2019

[6]Dutta P., Kumaravel A.,A novel approach to trust based identification of leaders in social networks,Indian Journal of Science and Technology,V-9,I-10,PP--,Y-2016

[7] Kumaravel A., Dutta P.,Application of Pca for context selection for collaborative filtering,Middle - East Journal of Scientific Research,V-20,I-1,PP-88-93,Y-2014

[8] Kumaravel A., Rangarajan K.,Constructing an automaton for exploring dynamic labyrinths,2012 International Conference on Radar, Communication and Computing, ICRCC 2012,V-,I-,PP-161-165,Y-2012

[9]P. Kavitha, S. Prabakaran "Adaptive Bilateral Filter for Multi-Resolution in Brain Tumor Recognition” International Journal of Innovative Technology and Exploring Engineering (IJITEE) ISSN: 2278-3075, Volume-8 Issue-8 June, 2019

[10] Kumaravel A.,Comparison of two multi-classification approaches for detecting network attacks, World Applied Sciences Journal,V-27,I-11,PP-1461-1465,Y-2013

[11]Tariq J., Kumaravel A.,Construction of cellular automata over hexagonal and triangular tessellations for path planning of multi-robots,2016 IEEE International Conference on Computational Intelligence and Computing Research, ICCIC 2016,V-,I-,PP--,Y-2017

[12] Sudha M., Kumaravel A.,Analysis and measurement of wave guides using poisson method,Indonesian Journal of Electrical Engineering and Computer Science, V-8,I-2,PP-546-548,Y-2017

[13] Ayyappan G., Nalini C., Kumaravel A.,Various approaches of knowledge transfer in academic social network,International Journal of Engineering and Technology,V-,I-,PP-2791-2794,Y-2017

[14] Kaliyamurthie, K.P., Sivaraman, K., Ramesh, S. Imposing patient data privacy in wireless medical sensor networks through homomorphic cryptosystems 2016, Journal of Chemical and Pharmaceutical Sciences92.

[15] Kaliyamurthie, K.P., Balasubramanian, P.C. An approach to multi secure to historical malformed documents using integer ripple transfiguration2016 Journal of Chemical and Pharmaceutical Sciences92.

[16]A.Sangeetha,C.Nalini,"Semantic Ranking based on keywords extractions in the web", International Journal of Engineering \& Technology, 7 (2.6) (2018) 290-292

[17] S.V.GayathiriDevi,C.Nalini,N.Kumar,"An efficient software verification using multi-layered software verification tool "International Journal of Engineering \& Technology, 7(2.21)2018 454-457

[18]C.Nalini,ShwtambariKharabe,"A Comparative Study On Different Techniques Used For Finger - Vein Authentication", International Journal Of Pure And Applied Mathematics, Volume 116 No. 8 2017, 327-333, Issn: 1314-3395

[19]M.S. Vivekanandan and Dr. C. Rajabhushanam, "Enabling Privacy Protection and Content Assurance in Geo-Social Networks", International Journal of Innovative Research in Management, Engineering and Technology, Vol 3, Issue 4, pp. 49-55, April 2018.

[20]Dr. C. Rajabhushanam, V. Karthik, and G. Vivek, "Elasticity in Cloud Computing”, International Journal of Innovative Research in Management, Engineering and Technology, Vol 3, Issue 4, pp. 104-111, April 2018.

[21] K. Rangaswamy and Dr. C. Rajabhushanamc, "CCN-Based Congestion Control Mechanism In Dynamic Networks", International Journal of Innovative Research in Management, Engineering and Technology, Vol 3, Issue 4, pp. 117-119, April 2018.

[22] Kavitha, R., Nedunchelian, R., "Domain-specific Search engine optimization using healthcare ontology and a neural network backpropagation approach", 2017, Research Journal of Biotechnology, Special Issue 2:157-166

[23] Kavitha, G., Kavitha, R., "An analysis to improve throughput of high-power hubs in mobile ad hoc network" , 2016, Journal of Chemical and Pharmaceutical Sciences, Vol-9, Issue-2: 361-363

[24] Kavitha, G., Kavitha, R., "Dipping interference to supplement throughput in MANET" , 2016, Journal of Chemical and Pharmaceutical Sciences, Vol-9, Issue-2: 357-360

[25] Michael, G., Chandrasekar, A.,’Leader election based malicious detection and response system in MANET using mechanism design approach", Journal of Chemical and Pharmaceutical Sciences(JCPS) Volume 9 Issue 2, April - June 2016 .

[26] Michael, G., Chandrasekar, A.,'Modeling of detection of camouflaging worm using epidemic dynamic model and power spectral density", Journal of Chemical and Pharmaceutical Sciences(JCPS) Volume 9 Issue 2, April June 2016

[27]Pothumani, S., Sriram, M., Sridhar, J., Arul Selvan, G., Secure mobile agents communication on intranet,Journal of Chemical and Pharmaceutical Sciences, volume 9, Issue 3, Pg No S32-S35, 2016

[28] Pothumani, S., Sriram, M., Sridhar , Various schemes for database encryption-a survey, Journal of Chemical and Pharmaceutical Sciences, volume 9, Issue 3, Pg NoS103-S106, 2016

[29] Pothumani, S., Sriram, M., Sridhar, A novel economic framework for cloud and grid computing, Journal of Chemical and Pharmaceutical Sciences, volume 9, Issue 3, Pg No S29-S31, 2016

[30]Priya, N., Sridhar, J., Sriram, M. "Ecommerce Transaction Security Challenges and Prevention Methods- New Approach” 2016 ,Journal of Chemical and Pharmaceutical Sciences, JCPS Volume 9 Issue 3.page no:S66-S68

[31]Priya, N.,Sridhar,J.,Sriram, M."Vehicular cloud computing security issues and solutions" Journal of Chemical and Pharmaceutical Sciences(JCPS) Volume 9 Issue 2, April - June 2016

[32] Priya, N., Sridhar, J., Sriram, M. "Mobile large data storage security in cloud computing environment-a new approach" JCPS Volume 9 Issue 2. April - June 2016

[33] Anuradha.C, Khanna.V, "Improving network performance and security in WSN using decentralized hypothesis testing "Journal of Chemical and Pharmaceutical Sciences(JCPS) Volume 9 Issue 2, April - June 2016

[34] Anuradha.C, Khanna.V, "A novel gsm based control for e-devices“ Journal of Chemical and Pharmaceutical Sciences(JCPS) Volume 9 Issue 2, April June 2016

[35] Anuradha.C, Khanna.V, "Secured privacy preserving sharing and data integration in mobile web environments " Journal of Chemical and Pharmaceutical Sciences(JCPS) Volume 9 Issue 2, April - June 2016.

[36] Sundarraj, B., Kaliyamurthie, K.P. Social network analysis for decisive the ultimate classification from the ensemble to boost accuracy rates 2016 International Journal of Pharmacy and Technology

[37] Sundarraj, B., Kaliyamurthie, K.P. A content-based spam filtering approach victimisation artificial neural networks 2016 International Journal of Pharmacy and Technology83.

[38] Sundarraj, B., Kaliyamurthie, K.P. Remote sensing imaging for satellite image segmentation 2016 International Journal of Pharmacy and Technology 8 .

[39] Sivaraman, K., Senthil, M. Intuitive driver proxy control using artificial intelligence 2016 International Journal of Pharmacy and Technology84.

[40] Sivaraman, K., Kaliyamurthie, K.P. Cloud computing in mobile technology 2016 Journal of Chemical and Pharmaceutical Sciences92.

[41] Sivaraman, K., Khanna, V. Implementation of an extension for browser to detect vulnerable elements on web pages and avoid click jacking 2016 Journal of Chemical and Pharmaceutical Sciences92 


\section{AUTHORS PROFILE}

A.Rama Assistant Professor, Department of Information Technology, Bharath Institute of Higher Education and Research, Chennai, India

Dr.A.Kumaravel Professor, Department of Information Technology, Bharath Institute of Higher Education and Research, Chennai, India

Dr. C. Nalini Professor, Department of Computer Science \& Engineering, Bharath Institute of Higher Education and Research, Chennai, India 